\title{
Interferon-y Autoantibodies as Predisposing Factor for Nontuberculous Mycobacterial Infection
}

\section{Florent Valour, Thomas Perpoint, Agathe Sénéchal, Xiao-Fei Kong, Jacinta Bustamante, Tristan Ferry, Christian Chidiac, Florence Ader, on behalf of the Lyon TB study group}

Author affiliations: Hospices Civils de Lyon, International Center for Research in Infectiology, Université Claude Bernard Lyon 1, INSERM U1111, Lyon, France (F. Valour, T. Perpoint, A. Sénéchal, T. Ferry, C. Chidiac, F. Ader); Necker-Enfants Malades Hospital, INSERM, Paris Descartes University, Paris, France

(J. Bustamante); St. Giles Laboratory of Human Genetics of Infectious Diseases, Rockefeller University, New York, New York, USA (X.-E. Kong, J. Bustamante)

\section{DOI: http://dx.doi.org/10.3201/eid2206.151860}

To the Editor: Recent advances in the understanding of antimycobacterial immune response have led to descriptions of predisposing conditions to dissemination of nontuberculous mycobacteria (NTM) infection. The interferongamma (IFN- $\gamma$ )/interleukin-12 (IL-12) axis is a critical pathway for intracellular killing of mycobacteria (online Technical Appendix Figure, http://wwwnc.cdc.gov/EID/ article/22/6/15-1860-Techapp.pdf) (1).We report a case of disseminated NTM infection in a woman from Laos who showed IFN- $\gamma$ autoantibodies. We also conducted a literature review to review similar cases.

A previously healthy 50 -year-old woman from Laos was referred to Hospices Civils de Lyon, France, for fever and generalized lymphadenopathies; pathological examination revealed a nonnecrotizing granuloma. Culture yielded Mycobacterium fortuitum. After a 3-month azithromycin/ciprofloxacin regimen, symptoms resolved. A few months later, a computed tomography scan showed persistence of enlarged mediastinal lymph nodes; biopsy disclosed nonspecific sinus histiocytosis. Mycobacterial cultures were negative. One month later, the patient reported intense dorsal spine pain. She was diagnosed with multifocal vertebral osteomyelitis, and tissue specimens tested positive for M. intracellulare. Azithromycin/ciprofloxacin was prescribed again. At 6 months, lesions worsened, and epidural abscess led to spinal cord compression, requiring decompressive laminectomy. Postoperative cultures were positive for $M$. intracellulare. Treatment was changed to azithromycin, rifabutin, ethambutol, and amikacin for 2 weeks, then rifabutin and moxifloxacin, which led to clinical improvement.
The patient experienced 2 episodes of thoracic herpes zoster concomitantly to each episode of illness. The recurrent NTM infection led us to investigate her immune status. Results of standard immunologic tests (leukocyte, serum level of immunoglobulin isotypes, and T-, B-, and NK0cell counts) and in vitro T-cell proliferation were normal; HIV testing was negative. Given its critical role in Mycobacteria clearance by host cells, we used IFN- $\gamma$ whole-blood activation to investigate further. IFN- $\gamma$ plasma level was undetectable, excluding complete forms of IFN- $\gamma$ R1/R2. IL-12 p40 subunit plasmatic level was decreased, but the IL-12 receptor was present and functional. In addition, both BCG and IL-12 stimulations failed to trigger IFN- $\gamma$ production. Ultimately, the detection of IFN- $\gamma$ autoantibodies in high levels explained these abnormalities. Antimycobacterial treatment was continued for 2 years, then changed to azithromycin suppressive therapy; IFN- $\gamma$ autoantibodies remained positive at the time of this report, 2 years after azithromycin initiation.

We reviewed the literature and found 63 other cases of IFN- $\gamma$ autoantibody-related NTM infection with sufficient detail to provide clinically relevant material for optimizing disease management (Table; online Technical Appendix). These cases overwhelmingly occurred among Asian populations (92.2\%) (2). Disease incidence may be underestimated, as suggested by large studies describing Asian patients with disseminated NTM infection without evidence of impaired immunity but for whom the IL-12/IFN- $\gamma$ axis was not investigated (3). This acquired autoantibodies-mediated immunodeficiency is more frequent among women, in whom the disease typically manifests in the second half of adult life (median 48 [IQR 44.8-60.0] years of age). Reported female:male sex ratios are 23/36 in Asian-born ( $\mathrm{n}=$ $59)$ and $4 / 1$ in non-Asian $(n=5)$ case-patients, respectively. Mechanism of the disease is briefly discussed in the online Technical Appendix.

M. avium complex predominated in the literature review, accounting for $40.4 \%$ of cases, followed by $M$. abscessus (31.6\%) and M. fortuitum (12.3\%). Infections were mostly multifocal, affecting lymph nodes $(\mathrm{n}=51$, $79.7 \%)$, osteoarticular tissue $(\mathrm{n}=37,57.8 \%)$, lungs $(\mathrm{n}=$ $30,46.9 \%)$, and skin and/or soft tissues $(\mathrm{n}=24,37.5 \%)$. Aside from NTM infections, other opportunistic infections were reported in $39(75.0 \%)$ patients, mostly Herpesviridae reactivations $(44.2 \%)$ and Salmonella infections $(25.0 \%)(3-6)$.

Specific treatment for IFN- $\gamma$ autoantibodies-associated NTM infection is not codified and required prolonged, multiple-drug regimens. The median treatment duration for the studies we reviewed was 31 (IQR 22.8-60.0) months. In some studies, clinicians used IFN- $\gamma$ administration (5 patients, 1 of whom was cured), but treatment likely was invalidated by the autoimmune-driven inhibitory 
Table. Summary of clinically relevant characteristics of 64 casepatients with IFN-y autoantibody-related nontuberculous mycobacterial infection found in literature review*

\begin{tabular}{|c|c|}
\hline Characteristics & Value \\
\hline \multicolumn{2}{|l|}{ Demographics } \\
\hline Female sex & $40(62.5)$ \\
\hline Median age, y (IQR) & $48.0(44.8-60.0)$ \\
\hline Asian origin & $59(92.2)$ \\
\hline Other autoimmune diseases & $7 / 27(25.9)$ \\
\hline \multicolumn{2}{|l|}{ Etiologic agents } \\
\hline Mycobaterium avium & $23(40.4)$ \\
\hline M. abscessus & $18(31.6)$ \\
\hline M. fortuitum & 7 (12.3) \\
\hline M. tuberculosis & $6(10.5)$ \\
\hline Other opportunistic infections & $39 / 52(75.0)$ \\
\hline Herpesviridae reactivations & 23/52 (44.2) \\
\hline Salmonella spp. & $13 / 52(25.0)$ \\
\hline $\begin{array}{l}\text { Median duration of intensive-phase } \\
\text { therapy, mo (IQR) }\end{array}$ & $31.0(22.8-60.0)$ \\
\hline$\geq 6$ & $31 / 31(100)$ \\
\hline$\geq 12$ & 29/31 (93.5) \\
\hline$\geq 18$ & 27/31 (87.1) \\
\hline$\geq 24$ & 23/31 (74.2) \\
\hline $\begin{array}{l}\text { Long-term antimicrobial suppressive } \\
\text { therapy }\end{array}$ & $3 / 30(10.0)$ \\
\hline Immunomodulatory therapies & $10 / 30(33.3)$ \\
\hline Rituximab & $6 / 30(20.0)$ \\
\hline IFN- $\gamma$ & $5 / 30(16.7)$ \\
\hline Intravenous immunoglobulins & $2 / 30(6.7)$ \\
\hline Plasmapheresis & $2 / 30(6.7)$ \\
\hline Cyclophosphamid & $1 / 30(3.3)$ \\
\hline \multicolumn{2}{|l|}{ Outcome } \\
\hline Cure & 21/62 (33.9) \\
\hline Improvement & $6 / 62(9.7)$ \\
\hline Relapse/persistence & 29/62 (46.8) \\
\hline Death & $6 / 62(9.7)$ \\
\hline \multicolumn{2}{|c|}{$\begin{array}{l}\text { *Values are no. (\%) except as indicated. Only articles with sufficient detail } \\
\text { were analyzed. A complete listing of articles reviewed from the literature is } \\
\text { provided in the online Technical Appendix Table } \\
\text { (http://wwwnc.cdc.gov/EID/article/22/6/15-1860-Techapp1.pdf). IQR, } \\
\text { interquartile range; IFN- } \gamma \text {, interferon gamma. } \\
\text { †Denominators indicate number of case-patients for which category of } \\
\text { data was available. }\end{array}$} \\
\hline
\end{tabular}

activity $(5,2,7)$. Other strategies included intravenous immunoglobulin $(\mathrm{n}=2)$, plasmapheresis $(\mathrm{n}=2)$, and cyclophosphamide $(\mathrm{n}=1)(7-9)$. The use of rituximab, a chimeric anti-CD20 monoclonal antibody targeting B-cells, has been recently associated with clinical response and decrease in IFN- $\gamma$ autoantibody levels as well as neutralizing ability $(6,7)$.

Final outcome was available for 56 patients who completed the intensive treatment phase; 21 (37.5\%) were declared cured. Six (10.7\%) patients died, and $29(51.8 \%)$ had persistent or relapsing infections. At the time of this report, additional patients were still being treated and showed improvement of symptoms. Despite this high rate of failure, long-term antimicrobial drug suppressive therapy has rarely been proposed as a causal factor. The origin of the case we report was related to the use of azithromycin suppressive therapy, similarly to disseminated NTM disease prophylaxis in HIV-infected patients before the era of highly active antiretroviral therapies (10), assuming the risk/ benefit balance including the possibility of NTM macrolide-resistant strain selection.

IFN- $\gamma$ autoantibodies are evidence of acquired immunodeficiency that should be considered in cases of unexplained disseminated NTM infections in Asian-born persons. Use of immunomodulation strategies is still debated, and long-term suppressive treatment should be considered for persisting high levels of neutralizing antibodies.

\section{Acknowledgments}

We thank Lyon tuberculosis study group members F. Ader, F. Biron, A. Boibieux, A. Bouaziz, E. Braun, G. Catho, N. Charhon, C. Chidiac, W. Chumbi-Flores, S. Couraud, G. Devouassoux, O. Dumitrescu, S. Ernesto, T. Ferry, D. Floret, N. Freymond, S. Gardes, S. Gerbier-Colomban, Y. Gillet, S. Goutelle, J. Grando, R. Grima, L. Hees, J. Karsenty, L. Kiakouama-Maleka, G. Lina, J. M. Maury, P. Miailhes, P. Nesme, T. Perpoint, E. Perrot, A. S. Ronnaux-Baron, S. Roux, J. Saison, A. Senechal, P. J. Souquet, H. Thai Van, F. Tronc, F. Valour, and P. Vanhems for choosing research field priorities and editing one another's reports for this article.

\section{References}

1. Wu U-I, Holland SM. Host susceptibility to non-tuberculous mycobacterial infections. Lancet Infect Dis. 2015;15:968-80. http://dx.doi.org/10.1016/S1473-3099(15)00089-4

2. Kampmann B, Hemingway C, Stephens A, Davidson R, Goodsall A, Anderson S, et al. Acquired predisposition to mycobacterial disease due to autoantibodies to IFN- $\gamma$. J Clin Invest. 2005;115:2480-8. http://dx.doi.org/10.1172/JCI19316

3. Browne SK, Burbelo PD, Chetchotisakd P, Suputtamongkol Y, Kiertiburanakul S, Shaw PA, et al. Adult-onset immunodeficiency in Thailand and Taiwan. N Engl J Med. 2012;367:725-34. http://dx.doi.org/10.1056/NEJMoa1111160

4. Chi C-Y, Chu C-C, Liu J-P, Lin C-H, Ho M-W, Lo W-J, et al. Anti-IFN- $\gamma$ autoantibodies in adults with disseminated nontuberculous mycobacterial infections are associated with HLA-DRB1*16:02 and HLA-DQB1*05:02 and the reactivation of latent varicella-zoster virus infection. Blood. 2013;121:1357-66. http://dx.doi.org/10.1182/blood-2012-08-452482

5. Döffinger R, Helbert MR, Barcenas-Morales G, Yang K, Dupuis S, Ceron-Gutierrez L, et al. Autoantibodies to interferon-gamma in a patient with selective susceptibility to mycobacterial infection and organ-specific autoimmunity. Clin Infect Dis. 2004;38:e10-4. http://dx.doi.org/10.1086/380453

6. Czaja CA, Merkel PA, Chan ED, Lenz LL, Wolf ML, Alam R, et al. Rituximab as successful adjunct treatment in a patient with disseminated nontuberculous mycobacterial infection due to acquired anti-interferon- $\gamma$ autoantibody. Clin Infect Dis. 2014;58:e115-8. http://dx.doi.org/10.1093/cid/cit809

7. Browne SK, Zaman R, Sampaio EP, Jutivorakool K, Rosen LB, Ding L, et al. Anti-CD20 (rituximab) therapy for anti-IFN- $\gamma$ autoantibody-associated nontuberculous mycobacterial infection. Blood. 2012;119:3933-9. http://dx.doi.org/10.1182/ blood-2011-12-395707

8. Koya T, Tsubata C, Kagamu H, Koyama K, Hayashi M, Kuwabara K, et al. Anti-interferon-gamma autoantibody in a patient with disseminated Mycobacterium avium complex. J Infect Chemother. 2009;15:118-22. http://dx.doi.org/10.1007/ s10156-008-0662-8 
9. Baerlecken N, Jacobs R, Stoll M, Schmidt RE, Witte T. Recurrent, multifocal Mycobacterium avium-intercellulare infection in a patient with interferon-gamma autoantibody. Clin Infect Dis. 2009;49:e76-8. http://dx.doi.org/10.1086/605581

10. Havlir DV, Dubé MP, Sattler FR, Forthal DN, Kemper CA, Dunne MW, et al. Prophylaxis against disseminated Mycobacterium avium complex with weekly azithromycin, daily rifabutin, or both. California Collaborative Treatment Group. N Engl J Med. 1996;335:392-8. http://dx.doi.org/10.1056/ NEJM199608083350604

Address for correspondence: Florent Valour, Department of Infectious Diseases, Hospices Civils de Lyon, 103 Grande-Rue de la Croix-Rousse, 69004 Lyon, France; email: florent.valour@chu-lyon.fr

\section{Loss of 89K Pathogenicity Island in Epidemic Streptococcus suis, China}

\section{Xiaolu Shi, ${ }^{1}$ Huiyan Ye, ${ }^{1}$ Jun Wang, Zhencui Li, Jingzhong Wang, Baoshan Chen, Ronghui Wen, Qinghua Hu, Youjun Feng}

\begin{abstract}
Author affiliations: Zhejiang University School of Medicine, Hangzhou, China (X. Shi, H. Ye, J. Wang, Z. Li, Y. Feng); Shenzhen Centre for Disease Control and Prevention, Shenzhen City, China (X. Shi, J. Wang, Q. Hu); Guangxi University, Nanning City, China (H. Ye, J. Wang, B. Chen, R. Wen)

DOI: http://dx.doi.org/10.32032/eid2206.152010
\end{abstract}

To the Editor: Streptococcus suis serotype 2 (SS2) is a previously neglected, newly emerging human pathogen that causes occupational and opportunistic infections $(1,2)$. Outbreaks of fatal human SS2 infections in China, featuring streptococcal toxic shock syndrome, in 2005 seriously challenged global public health (3-5). The epidemic strain is unusual in that it contains a unique $89-\mathrm{kb}(89 \mathrm{~K})$ pathogenicity island (PAI) $(3,6,7)$. We observed the loss of genes from the $89 \mathrm{~K}$ PAI in sporadic cases in southern China in 2007, implying the dynamic evolution of this PAI (8). Therefore, 89K PAI might be able to be used to monitor prevalent strains of $S$. suis in China (8).

We report 10 recurrent cases of human $S$. suis infections during 2008-2015 in southern China. Most of the hospitalized patients were male workers in close contact with pigs, pork products, or both. These patients typically exhibited clinical syndromes of meningitis, including headache, coma, vomiting, and fever. The bacterial strains acquired from humans were as follows: 2 isolates in 2008 (Stre08001 and Stre08002), 2 in 2009 (Stre09001 and Stre09002), 2 in 2011 (Stre11001 and Stre11002), 3 in 2013 (Stre13002,

${ }^{1}$ These authors contributed equally to this article.
Stre13003, and Stre13004), and 1 in 2015 (Stre15001). Microbial and molecular assays proved that these clinical isolates were $S$. suis (online Technical Appendix Figures 1, 2, http://wwwnc.cdc.gov/EID/article/22/6/15-2010Techapp1.pdf). Multiplex PCR-based molecular determination (16S rDNA, mrp, epf, and cps-2j) suggested that all strains except Stre13002 were SS2 (online Technical Appendix Figure 2) (3). To determine whether these clinical isolates derived from the same Chinese epidemic clone $05 Z Y H 33$, we sequenced an array of virulence factor-encoding genes, as well as the $16 \mathrm{~S} \mathrm{rDNA}$ genes. Phylogenetic trees indicated that all 10 clinical strains are classified into the same subclade as that of the strain 05ZYH33 (online Technical Appendix Figure 2).

Subsequent analyses by pulsed-field gel electrophoresis revealed that genotypes are diverse among these clinical strains, which can be roughly divided into 6 groups (online Technical Appendix Figure 3). Given that the Sao surface antigen protein possesses 3 allelic variants (Sao-L [670 aa], Sao-M [580 aa], and Sao-S [489/490 aa]) (9), we thus assayed it with these clinical strains. Unexpectedly, we found 2 more new allelic variants, referred to as Sao-L1 (640 aa) and Sao-L2 (611 aa). Except for the strain BM407, which is a Chinese epidemic SS2 encoding Sao-L1, a version 30 residues shorter than Sao-L, 8 of the 10 clinical $S$. suis isolates consistently had the same new form of Sao protein, Sao-L2 (611 aa) (online Technical Appendix Figure 4).

Because 89K PAI is in dynamic evolution, determining whether it remains present in clinical strains is of interest. As previously designed (online Technical Appendix Table 1), a specific pair of boundary primers (1/6) was applied for PCR-based detection of the 89K PAI (Figure, panel A, http://wwwnc.cdc.gov/EID/article/22/6/152010-F1.htm). In principle, the PCR-positive result suggests the absence of $89 \mathrm{~K} \mathrm{PAI}$, whereas the PCR-negative result indicates the presence of $89 \mathrm{~K}$ PAI $(6,8)$. Unlike the epidemic strain 05ZYH33 that has the 89K PAI, 9 of the 10 clinical strains examined (Stre08001, Stre08002, Stre09001, Stre09002, Stre11001, Stre11002, Stre13002, Stre13003, and Stre13004) were unexpectedly found to be PCR positive for the unique 1/6 DNA fragment with expected size of $\approx 1.5 \mathrm{~kb}$ (Figure, panel $\mathrm{B}$ ). This finding indicates that the $89 \mathrm{~K}$ PAI is lost in these 9 clinical strains. We saw similar scenarios in the subsequent PCRs for other inner genes/DNA fragments (943 and 944 [10]; 1/2, 3/4, and $5 / 6$ [8]) inside of 89K PAI (Figure, panel C). Further DNA sequencing of the $1 / 6 \mathrm{PCR}$ product showed that it matches well with the 2 boundary regions neighboring the $89 \mathrm{~K}$ PAI, validating the loss of $89 \mathrm{~K} \mathrm{PAI}$ in these 9 clinical isolates (Figure, panel D). In contrast, the strain Stre15001 behaved similarly to that of the $05 \mathrm{ZYH} 33$ containing the $89 \mathrm{~K}$ PAI, in that both are PCR positive for the 4 amplicons 